\section{$\underset{\substack{\text { hommes } \\ \text { \& migrations }}}{ }$}

\section{Hommes \& migrations}

Revue française de référence sur les dynamiques

migratoires

1286-1287 | 2010

Les migrations subsahariennes

\title{
Les mères, les filles et la cuisine "africaine" en situation de migration (Marseille)
}

Analyse comparative (1998-2010)

Julie Lioré

\section{(2) OpenEdition}

Journals

Édition électronique

URL : https://journals.openedition.org/hommesmigrations/1746

DOI : 10.4000/hommesmigrations. 1746

ISSN : 2262-3353

Éditeur

Musée national de l'histoire de l'immigration

Édition imprimée

Date de publication : 1 juillet 2010

Pagination : 178-188

ISSN : 1142-852X

Référence électronique

Julie Lioré, "Les mères, les filles et la cuisine "africaine" en situation de migration (Marseille) », Hommes \& migrations [En ligne], 1286-1287 | 2010, mis en ligne le 29 mai 2013, consulté le 08 décembre 2022. URL : http://journals.openedition.org/hommesmigrations/1746 ; DOI : https:// doi.org/10.4000/hommesmigrations. 1746 


\section{Les mères, les filles et la cuisine "africaine" en situation de migration (Marseille) Analyse comparative (1998-2010)}

Par Julie Lioré, anthropologue, chercheure associée au laboratoire d'anthropologie bioculturelle (UMR 6578 - Marseille) et au programme de recherche AlimAdos (ANR/Cniel-OCHA)

Soumises aux sollicitations multiples de modes de consommation standardisés, par manque de temps ou de volonté, les adolescentes issues de familles ouest-africaines savent de moins en moins préparer les plats de leurs aînées. Pourtant les mères s'efforcent de transmettre à leurs filles la richesse de leur patrimoine culinaire. En une dizaine d'années, cette désaffection qui continue de gagner du terrain laisse entrevoir un renouvellement des pratiques. Dans leurs attitudes vis-à-vis de la cuisine "africaine", ces jeunes femmes éclairent la manière dont elles façonnent leur identité. 
La transmission demeure l'instrument par excellence de la continuité sociale, la "composante active de ce qui, de manière institutionnelle ou informelle, se communique à l'intérieur d'une communauté humaine ${ }^{(1)}$ ". Toutefois, il s'agit là d'un patrimoine soumis, avec le passage des générations, à des déperditions, réinterprétations et recompositions, plus encore dans les groupes confrontés à des ruptures ou à des mutations ${ }^{(2)}$. Il est avéré aujourd'hui que le concept de "tradition" ne peut plus être envisagé comme une totalité se reproduisant à l'identique de génération en génération, "mais capable d'engendrer de la création ou de la recréation, qui relie entre elles les générations et fabrique leur devenir(3)". C'est dans cette perspective aussi qu'il est urgent de "réhabiliter la part de l'enfant dans le processus d'apprentissage, en montrant quel rôle il y joue et d'où vient qu'il puisse le jouer (4)". En effet, les recherches dans le domaine de la cognition montrent que l'enfant apprendrait moins ce qu'il sait des adultes que par ses propres capacités et sa contribution pour y parvenir.

La transmission des savoir-faire culinaires et alimentaires, comme les représentations, les pratiques et les préférences du même registre, sont culturellement construits et demeurent profondément liés aux parcours sociaux, familiaux, migratoires, mais aussi et surtout personnels. Compte tenu des situations aux références culturelles et sociales multiples - comme le cas des enfants dont les parents ou les grands-parents ont migré d'un pays vers un autre - la tradition n'est en effet plus ce qu'elle était ${ }^{(5)}$.

Grâce à un travail sur le long terme ${ }^{(6)}$ avec des familles ouest-africaines à Marseille, il s'agit d'explorer les modes de transmission et les modalités d'apprentissage dans la durée pour comprendre comment s'opère la différence parents/enfants, à travers le rapport des seconds à la culture des premiers, et vice versa. Cette approche transversale sur douze ans croise les données et l'analyse d'une thèse de doctorat, dont l'étude de terrain a été menée entre 1998 et $2001^{(7)}$, et les données recueillies dans le cadre du programme de recherche AlimAdos ${ }^{(8)}$ entre 2008 et 2010.

La population sur laquelle reposent ces deux études comprend des parents nés en Afrique subsaharienne (Sénégal, Mali, Mauritanie), majoritairement musulmans et arrivés en France avant d'être parents, ou bien nés en France, ainsi que leurs descendants, pour la plupart nés en France. Compte tenu de "l'impossibilité de recourir à la catégorie non pertinente parce que non homogène de 'famille africaine $e^{(9) ", ~ n o u s ~ p r e ́ c i s e r o n s ~}$ qu'il s'agit principalement de familles soninké, wolof ou manjak, de confession musulmane ou chrétienne. La méthodologie employée a été qualitative : entretiens menés auprès des parents et de leurs enfants (ensemble ou séparément), repas pris et temps passé en famille, observations faites à domicile et à l'occasion de fêtes "traditionnelles ${ }^{(10) "}$ comme des mariages ou des baptêmes. 


\section{Un décalage d’âge entre la génération des mères et celle des filles}

Lors d'un échange à propos de transmission des savoir-faire culinaires à sa fille de 12 ans, Madame Hélène ${ }^{(11)}$ réagit : "Elle est encore un peu jeune pour ça !'. Puis elle s'interrompt et corrige : "Quoi que, je dis n'importe quoi parce que, moi, ma mère m'a appris à commencer à préparer, à faire du riz quand javais 11 ans." Madame Astou aussi, et comme beaucoup d'autres femmes de sa génération, "à 10 ans, 11 ans, déjà je savais cuisiner." Mères et filles tendent à expliquer ce décalage par le fait de vivre en France, un contexte où quantité de facteurs, comme la scolarité, la disponibilité, une sociabilité élargie, une éducation assouplie ne permettent plus cet apprentissage aussi précoce et complet qu'autrefois pour les mères ou en Afrique aujourd'hui.

Il y a dix ans, les filles, nées en France pour la plupart, percevaient ce décalage comme un retard, souvent vécu comme un complexe par rapport à leurs semblables en Afrique. Guethy (13 ans, terrain 1998/2001) affirme que, si elle avait grandi en Afrique: "Je saurais déjà faire la cuisine et tout, je serais un peu plus avancée. Il y en a, au Sénégal, qui ont 13 ans et qui savent faire plus de choses que moi." Quant à Khoumba (14 ans, terrain 1998/2001): "Moi je suis petite encore, mais ma soeur [adulte], elle sait faire". Si Khoumba avait grandi en Afrique, elle ne se considèrerait pas comme étant trop jeune pour apprendre et savoir préparer les principaux plats, ni le serait par sa famille et la société de référence. La

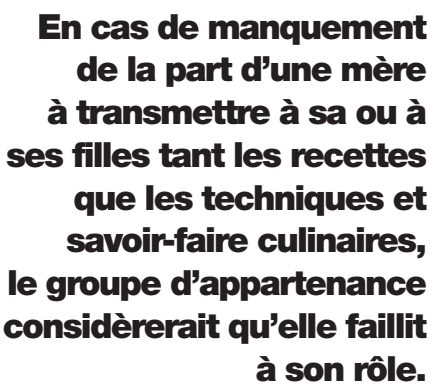

norme, en effet, prescrit que "n'importe quelle fille doit savoir cuisiner à 15 ans pour la famille" (Madame Marie, terrain 1998/2001). Autrement dit, à cet âge, cet apprentissage devrait être achevé.

Aujourd'hui, si la plupart des adolescentes de 15 ans savent préparer des plats "simples et vite faits", type steak-frites, pâtes, ou encore les omelettes, Mariama (15 ans) est la seule, parmi celles rencontrées de son âge, à savoir réaliser les

principaux plats de riz, et notamment le "riz rouge" (riz à la sauce tomate). À l'occasion d'une récente visite à la famille, Khanify, la mère de Mariama, félicite sa fille : "Elle a fait la sauce de poulet, elle a fait bien, jai dit bravo." Cette dernière a franchi, de manière informelle, le rite de passage qui fait d'elle une femme accomplie en préparant pour sa famille un "riz rouge parfait"(Khanify). En revanche, ces dernières années, la plupart des filles assument davantage d'apprendre plus tard, voire de ne pas encore savoir "correctement" cuisiner, notamment les plats relevant du registre culinaire "africain". 


\section{Un "devoir de fille"}

Savoir cuisiner est, en Afrique subsaharienne et encore aujourd'hui en situation de migration, une question d'accomplissement, de passage d'un groupe d'âge à une autre, autrement dit, de rang dans la société. Une jeune fille, en sachant cuisiner, est prête à devenir une épouse et une mère. "Tu peux trouver un mari africain maintenant!', se sont amusés un jour Madame Awa et son mari, leur fille leur ayant révélé qu'elle savait préparer certains plats "africains". Cette transmission est fondamentale pour les mères. D'une part, "parce que c'est la tradition" (Mme Yvette), "c'est la continuité, c'est les valeurs de tout ce qu'on aime" (Mme N'Dack). D'autre part, "une fille doit savoir cuisiner, c'est pour elle." (Mme Mariam). Par ailleurs, le savoir-faire culinaire est aussi envisagé sous un angle pratique : Comment elles vont faire après, si moi je ne leur apprends pas ? Elles vont apprendre comment?", s'inquiète Madame Yvette.

Du point de vue des jeunes filles, il y a dix ans comme aujourd'hui, cet apprentissage demeure indispensable. "C'est vrai que si on est mariée, c'est important qu'on sache." (Khoumba, 14 ans, terrain 1998/2001). Par-delà le contexte marital et familial, "c'est important pour moi plutôt", précise-t-elle, telle une pièce majeure du puzzle de sa personnalité. Guethy est tout aussi ferme : "Je ne vais pas rester jusqu'à 25 ans sans savoir faire un plat culinaire de mon pays quand même!'Ce serait déshonorant à cet âge, ainsi qu'un manquement, tant de sa part que de celle de sa mère, qui aurait failli dans son rôle de mère. Guethy ajoute: "Au Sénégal, quand on est une jeune fille, on doit apprendre à faire la cuisine."

Ces dernières années, les filles considèrent autant que leurs aînées l'importance de cet apprentissage, mais souvent, elles le projettent dans le futur plus qu'elles ne l'envisagent à court ou moyen terme. Par exemple, Absa C. (16 ans) explique : "J'ai toujours dit que plus tard, je voudrais apprendre, mais 'plus tard', jusqu'à quand je vais dire ça ? C'est ça le problème?' Autrement dit, l'écart se creuse encore. Pour Maguette (15 ans), "c'est important de savoir faire un jour", mais pas dans l'immédiat où elle s'attelle à d'autres tâches : scolarité, activités à l'extérieur... Dans leurs représentations, plus que dans les pratiques réelles pour certaines, apprendre à cuisiner reste une question d'accomplissement. Selon Fatou D. (18 ans), une fille ne sachant pas cuisiner, "ça sert à rien en gros, elle n'a pas accompli son devoir de fille. C'est important quand même je trouve, on n'a pas dit de devenir cuisinière experte, mais au moins faire quelque chose, c'est bien." Fatou est l'aînée de la fratrie qui compte une fillette de 6 ans et quatre garçons, deux facteurs qui peuvent être déterminant dans le cadre d'un processus d'apprentissage achevé. 


\section{Transmissions verticale et horizontale}

Par transmission verticale, entendons ce qui est transmis de mères en filles, mais aussi par les filles à leur mère. À l'horizontale, il s'agit des échanges de recettes et de leurs savoir-faire entre soeurs, cousines ou amies avec, en filigrane, l'influence de la publicité, de la restauration scolaire et, plus récemment, d'Internet et des sites de recettes en ligne de plus en plus nombreux et visités.

Le rôle des femmes de l'âge de la mère (tantes, amies) est de transmettre aux filles les précieux savoir-faire culinaires qui, comme les pratiques alimentaires, "sont encore loin d'être réduits à leur seule fonction nutritive mais sont plutôt souvent le centre de l'expression et de la transmission des rôles familiaux ${ }^{(12) . " ~ " Q u a n d ~ j e ~ p a r s ~ a u ~ S e ́ n e ́ g a l, ~ o n ~}$ m'apprend', explique Fama (13 ans) en ne désignant pas une personne en particulier. Ainsi, les occasions d'apprendre sont multipliées, une façon pour le groupe d'assurer, voire de renforcer l'apprentissage. Or, dans la migration et compte tenu, notamment, des conditions de vie et de logement qui ont eu pour effet de réduire les familles étendues en Afrique à des familles nucléaires en France, les mères se retrouvent seules dans ce rôle, qu'elles partagent néanmoins avec les filles aînées, qui transmettent aux cadettes ce qu'elles-mêmes ont appris. En cas de manquement de la part d'une mère à transmettre à sa ou à ses filles tant les recettes que les techniques et savoir-faire culinaires, le groupe d'appartenance considèrerait qu'elle faillit à son rôle et à ses obligations. Aussi vis-à-vis d'elle-même, se priverait-elle de son privilège de mère.

"Malheureusement, on n'a plus le temps de préparer pour nos enfants", déplore Madame Hélène dont les journées sont remplies par son travail, ses engagements associatifs, l'organisation des entraînements de sport de ses enfants, etc. Or, sans pratique, la transmission est compromise. Le travail salarié, la scolarité, les activités extra-familiales sont autant de freins à l'apprentissage des plats "typiques". Les filles apprennent encore aujourd'hui à cuisiner, mais "des choses simples et vite faites”(Fatou C., 18 ans). Les filles apprennent à cuisiner les "plats simples", voire "le riz" avec leur mère, désormais seule et isolée dans ce rôle. En revanche, elles ont aujourd'hui accès à une kyrielle de moyens d'apprentissage culinaire, devenus impersonnels et informels : Internet, livres de recettes ${ }^{(13)}$ fournis avec l'achat d'un appareil ménager, chaînes de télévision câblées spécialisées dans la gastronomie, émissions télévisées qui déclinent jeux et expériences culinaires en tous genres. Aussi, "si la réceptivité des destinataires est défaillante, le modèle transgénérationnel peut alors basculer vers l'intragénérationnel, voire même vers une autoformation qui tend à s'émanciper des héritages ${ }^{(14)}$ ".

En pleine période d'expérimentation, les filles n'hésitent pas à innover, tandis que leurs mères, pour la plupart, perpétuent avant tout leur propre répertoire culinaire, ce qui n'exclue pas d'y intégrer des recettes dites "françaises", souvent basiques. "Des 
fois jimagine des choses, la dernière fois, jai pris des pâtes, jai mis du poivron, un peu de tout ce que jai trouvé", rapporte Fama (13 ans). Les influences de la restauration scolaire ne sont pas sans transformer les habitudes alimentaires des familles.

Les aînées continuent de jouer un rôle dans la transmission des différents savoir-faire auprès de leurs cadettes, des savoirs qu'elles-mêmes ont appris avec leur mère ou leurs surs plus âgées, leurs cousines ou leurs amies. "Ceest de ses aînés immédiats qu [un cadet] apprendra, beaucoup plus que de ses parents ${ }^{(15)}$." Toutefois, les contenus transmis dans le domaine culinaire et alimentaire se sont transformés. Dorénavant, les aînées apprennent aux cadettes à préparer des plats "simples et vite faits" ou "français", autrement dit, à faire cuire des pâtes, un steak haché, ou encore à préparer un gratin.

\section{Modalités d’apprentissage}

Les modalités de transmission - "transmises de préférence par voie de participation. On demande donc implicitement à l'enfant d'observer, puis d'imiter, de s'exercer, d'essayer par luimême ${ }^{(16) "}$ - demeurent identiques depuis l'époque où les mères apprenaient en Afrique. Par étapes successives, "ma mère, elle m’appelle parfois pour éplucher" (Fama, 13 ans), "j’aide, je vois et tout, je commence à mémoriser" (Fatou D., 18 ans). À terme, "je 
fais pour savoir si jai bien compris" (Mariama, 15 ans). Autrement dit, "elle me montre comment on coupe les oignons, comment on coupe la viande, comment on fait revenir, quand il faut mettre le sel. Il y a un temps pour faire revenir la viande ou les oignons, chaque chose en son temps... elle surveille deux ou trois fois et après elle me laisse une fois toute seule et après je prends l'habitude", explique Fatou D. (18 ans) de manière détaillée. En effet, "dans beaucoup d'apprentissages, la part verbalisée semble réduite ${ }^{(17), " ~ " p l u s ~ p a r ~ i m p r e ́ g n a t i o n ~ q u e ~}$ par apprentissage explicite ${ }^{(18)}$. Si les modalités de transmission demeurent, le contenu de ce qui est transmis connaît de profondes mutations. Les mères apprennent aux filles des plats "simples", autrement dit "français". Madame Mariam explique, à propos de Fama, sa fille âgée de 13 ans : "Des fois, elle fait des pâtes, des omelettes, steaks, des choses simples quoi, mais elle commence. Le riz, je lui montre mais pas toujours." En effet, "choses simples" et "riz" font partie de deux registres bien distincts. Aujourd'hui, plus encore qu'il y a dix ans en arrière, les mères apprennent aux filles à "se débrouiller", et moins à préparer le riz et les différentes "sauces" qui l'accompagnent.

\section{Des filles pas toujours motivées}

Madame Mariam raconte: "Moi je voulais pas faire la cuisine, jaimais pas. Ma tante, elle m'a appris, elle m'a forcée, elle me tapait pour le faire quoi, parce que jaimais pas du tout, mais à force, elle m'a appelée souvent, et j'ai su faire..." Aujourd'hui, comme il y a dix ans, les filles ne sont plus obligées de cuisiner au point d'être battues pour apprendre et pratiquer. "Je suis pas obligée! Des fois je regarde ma mère, après, quand elle est pas là, qu'elle rentre un peu tard et ben je le fais, mais ma mère, elle moblige pas, si je veux le faire, je le fais", explique Guethy (13 ans, terrain 1998/2001). L'obligation collective a fait place à un choix individuel. Une décision quelquefois liée à une situation nouvelle, notamment conjugale. À propos de l'une de ses filles, Madame N'Dack (terrain 1998/2001) rapporte: "Elle s'est toujours débattue pour pas... elle me disait, moi, je vais avoir quelqu'un qui va me faire la cuisine et c'est elle qui a le plus fait de cuisine africaine dès qu'elle s'est mise avec son copain, il mange la cuisine africaine... Et après, 'maman, tu me montres?', elle a vite appris..."

Toutes les filles de plus de 15 ans savent préparer un repas simple. En revanche, rares sont celles qui savent cuisiner un mafé ou un yassa, et aucune le tieboudien ${ }^{(19)}$. Fatou D. (18 ans), pourtant expérimentée en matière de cuisine africaine, reconnaît : "Le tieb', non, je sais pas le faire, il est compliqué, ça prend du temps." Ou encore, "ça non, c'est trop dur! Je sais ce qui faut mettre comme ingrédients, mais la sauce, c'est dur" (Guethy, 13 ans, terrain 1998/2001). Toutes apprécient le tieb', comme les autres plats africains, et comptent sur leur mère pour en manger à l'occasion. 
Les arguments des adolescentes sont, en général, le manque de patience, d'intérêt ou de temps, et surtout le choix de faire autre chose. Fatou C. (18 ans) explique : Elle [sa mère] nous dit à chaque fois, 'venez regarder comme je fais!', mais en fait, on n'a pas la patience, c'est trop long." Sa soeur Absa (16 ans) ajoute : "Ça m'intéresse pas vraiment." Je lui demande si elle aurait envie d'apprendre, dans l'absolu, Absa répond : "Enfin si, mais faut être patient, c'est ça le problème, et je suis pas patiente, jai autre chose à faire." Madame Astou, la mère de Fatou et d'Absa, confirme : "Eux, ils préfèrent le manger qu'on fait rapidement. Nous, on prend le temps, le manger, on le fait doucement, pendant 2 heures, 3 heures. Mes enfants, ils ont pas la patience comme ça."

Dorénavant, les aînées apprennent aux cadettes à préparer des plats "simples et vite faits" ou "français", autrement dit, à faire cuire des pâtes, un steak haché, ou encore à préparer un gratin. À 12 ans, Dieneba répond "pas trop...", suivi d'une moue, lorsque je lui demande si elle a envie d'apprendre à cuisiner. Sa mère confirme : "Elle est pas du tout motivée", pourtant, "la cuisine est essentielle chez nous, c'est le lien même, c'est lui apprendre quand elle veut bien apprendre..." Dieneba est encore jeune, sa mère ne renonce pas.

Le plus souvent, notamment par manque de temps durant la semaine, les mères préparent "le riz" le week-end. Or, le samedi matin, les adolescentes sont soit au lycée ou à l'école coranique, soit "elles se lèvent tard, avant que jai fini le manger", déplore Madame Astou. Quant au samedi après-midi, elles préfèrent sortir avec leurs cousines ou leurs amies. "Ma mère, elle essaie de nous retenir à chaque fois, mais nous, on voudrait sortir avec nos copines." (Absa C., 16 ans). Seule Fatou D. (18 ans) considère cette responsabilité avec plus de maturité: "Moi jessaie de m’adapter aux deux situations : y a un temps pour sortir avec les copines et y a un temps pour cuisiner." La plupart des mères déplorent cette rupture annoncée. "Quand vous dites, venez, je vous montre, ils ont pas la patience, ils restent même pas un quart d'heure! [...] C'est dommage, mais comme ils sont nés ici, donc le manger français, peut-être, s'ils sont mariés ici, ilspeuvent vivre avec leur mari comme ça", se désole Madame Astou. Madame Khanify ironise à propos de sa fille Aminata, aujourd'hui âgée de 21 ans : "Comment tu vas faire pour manger? Tu vas aller au restaurant?' Quatrième d'une fratrie de six soeurs, Aminata, qui n'a jamais aimé cuisiner, "à la limite, les trucs vite faits", est en quelques sortes passée entre les mailles du filet de l'apprentissage. De même que dans les familles d'ouvriers agricoles immigrées dans le vignoble bordelais observées par Chantal Crenn, "de la place dans la fratrie et du nombre de soeurs dépend l'obligation de savoir cuisiner ${ }^{(20) !}$. Mères et filles font, pour certaines, le compromis suivant : pendant que la mère cuisine, les filles s'occupent du ménage. 
À propos de cette rupture et des mariages à venir, les filles s'interrogent. "On est la jeunesse qui est née en France, et on a cassé le... et voilà, on est pas bien, on veut savoir aussi, on veut apprendre mais c'est trop dur. Par exemple, il y a une année où on avait essayé moi et mes soeurs, on avait essayé de faire, ma soeur elle a raté après voilà, on a abandonné." (Absa C., 16 ans). Je demande à Absa ce que pensera, d'après elle, son futur mari d'une cuisine "vite faite", elle répond : "Cest ce qui me dit mon père! Il y aura que ça de toute façon, du surgelé et voilà, tant pis..." J'ai aussi posé la question à sa soeur aînée Fatou (18 ans): "Cest ce que mes parents me disent tout le temps, mais je dis ma grand-mère, elle a un restaurant sénégalais, il ira là-bas [rires] !' Elle poursuit, à propos de cuisine africaine : "J'ai remarqué que c'est plus long que les repas français et tout ça, faut attendre longtemps, attendre que ça mijote et tout ça, alors que les repas français, c'est plus vite fait."

\section{Le savoir-faire des filles}

La cuisine africaine, ou "manger africain ${ }^{(21)}$ ", est définie par les mères comme par leurs filles par opposition à la cuisine dite "française". Toutes l'évoquent, le plus souvent, en termes de "riz" ou de "sauces". "Ce qu'on appelle en Afrique de l'Ouest les 'sauces', c'està-dire les légumes, les graisses et les viandes qui fournissent à la fois un assaisonnement, des protéines et des liquides complémentaires, des vitamines et des sels minéraux indispensables ${ }^{(22)}$." La charge affective à l'égard du "riz" et de ses sauces est souvent bien plus manifeste que celle de la cuisine dite "française", associée à simplicité et à rapidité, autrement dit, "fonctionnelle". La cuisine familiale oscille souvent, dans le discours des adolescentes ${ }^{(23)}$, entre rapport affectif (cuisine de la mère valorisée ou défendue lors de stigmatisation) et critiques (cuisine trop grasse ou trop riche). Elle n'en demeure pas moins un support d'affirmation identitaire.

Ces sauces sont, le plus souvent, préparées à base de sauce tomate concentrée, d'oignons et de KUB OR, puis déclinées : tieboudien, mafé, yassa, soupokandia (gombos et huile de palme), etc. Ces sauces variées et différentes d'une région à l'autre, d'un groupe à l'autre, agrémentent du riz, cuit à l'étouffée ou à la vapeur, qui peut être "rouge" s'il est préparé avec de la sauce tomate, sinon "blanc" ou "cul nu", ainsi que du poisson, de la viande ou du poulet. La cuisine sahélienne, explique Bernard Maire, consiste en "un plat unique accompagné d'une sauce qui donne du goût autant qu'une bonne valeur nutritionnelle au plat ${ }^{24)}$." Quant au riz, il demeure dans la migration la base de l'alimentation.

Quelques-unes des filles ont appris à préparer certaines sauces, le plus souvent peu élaborées. "Le yassa, c'est le riz avec les oignons, oui, celui-là jarrive à le faire, le mafé, jarrive pas." (Fatou C., 18 ans). Ou encore "le pastel, c'est du poisson, du persil et une pâte, 
après on fait comme ça [Guethy, 13 ans (terrain 1998/2001) mime le geste qui permet de retourner la pâte], on prend la fourchette, sinon on prend un instrument, ça fait comme ça, pour faire arrondir." Quant au riz et à sa cuisson à la vapeur ou à même la sauce, la plupart des filles ne parviennent pas à le faire : "J'ai essayé, mais jai raté, le riz il était sec." (Fatou C., 18 ans).

Lorsqu'elles sont disponibles et motivées, les filles aident leur mère en cuisine et savent, comme Fama (13 ans), "mariner la viande, mettre sur le feu et tout ça". Mais souvent, comme Maguette (15 ans), "la sauce, je sais pas faire toute seule". Quoi qu'il en soit, c'est "pas comme ma mère", (Fama, 13 ans). Il y a dix ans, les adolescentes tendaient à valoriser l'alimentation africaine et à surestimer leurs propres savoir-faire. Aujourd'hui, cette alimentation est désormais perçue comme étant "plus grasse" que d'autres types d'alimentation, ou "trop riche", aussi la plupart des adolescentes refusent-elles d'en consommer trop régulièrement depuis quelques années. Quant aux savoir-faire, ils sont le plus souvent reconnus comme n'étant pas ou peu maîtrisés. Prétendre que l'on sait faire peut aussi être dû au processus même de l'apprentissage, dont les premières étapes consistent à observer, puis à progressivement expérimenter. À la question de savoir si elle sait préparer des plats "africains", Khoumba (14 ans, terrain 1998/2001) répondait : "Quoi, je sais le faire, mais je sais pas..."

\section{Conclusion}

Ces dix dernières années marquent une série de transformations dans le domaine de la transmission et de l'apprentissage des savoir-faire culinaires : différence d'âge, qui a sensiblement reculé entre la génération des mères et celle des filles, et différence de genre, où les garçons qui le souhaitent ne parviennent pas encore ni toujours à avoir une place dans la cuisine, un espace qui demeure féminin. Ces différents changements ont opéré un élargissement par certains aspects et une déperdition par d'autres. En effet, les répertoires culinaires, les contenus transmis, les habitudes alimentaires des familles se sont étendus ou transformés. Inversement, le réseau d'initiatrices, l'apprentissage spécifique des plats "africains", ou encore la motivation des filles pour apprendre se sont simplifiés ou affaiblis. L'obligation et les pratiques collectives d'autrefois font place aujourd'hui à un choix et à des comportements individuels. Toutefois, ces profondes mutations ne s'opèrent pas partout de manière homogène. Si le positionnement des parents demeure significatif dans l'analyse des différentes manières de faire familiales, l'impact sur les enfants n'est toutefois plus aussi établi ces dernières années. L'histoire personnelle, les choix et les ressentis font désormais la différence. En effet, "des valeurs de choix, de maîtrise de soi, de réalisation 
personnelle, ont supplanté celles des logiques de l'héritage", autrement dit, "les identités ne sont plus systématiquement héritées "25)" Les enfants, et plus encore les adolescentes, doivent, en revanche, composer aujourd'hui entre la contradiction de l'héritage avec la peur de trahir des manières de faire familiales et l'ambivalence entre cuisine familiale et cuisines au pluriel (cantine, pairs, régionale ou transnationale, etc.), lesquelles cohabitent désormais. Chacune, en alternance selon les contextes et les appétences, relève d'une logique d'affirmation identitaire personnelle.

\section{Notes}

1. Choron-Baix Catherine,"Transmettre et perpétuer aujourd'hui", in Ethnologie française, vol. $X X X, n^{\circ} 3,2000$, p. 357.

2. Voir Lenclud Gérard, "La tradition n'est plus ce qu'elle était...", in Terrain, n 9, 1987, pp. 110-123 ; Choron-Baix Catherine,"Transmettre et perpétuer aujourd'hui", in Ethnologie française, vol. XXX, n³, 2000, pp. 357-360 ;

Corbeau Jean-Pierre et Poulain Jean-Pierre, Penser l'alimentation : entre imaginaire et rationalité, Toulouse, Privat, 2002 ; Crenn Catherine, "Le tajine à la bouillie bordelaise. Transmission des manières de cuisiner dans les familles des ouvriers agricoles immigrées dans le vignoble bordelais", in Cahiers de l'OCHA, n 11 : Faire la cuisine, 2006, pp. 152-161.

3. Choron-Baix Catherine,"Transmettre et perpétuer aujourd'hui", op. cit., p. 359.

4. Lenclud Gérard, "Apprentissage culturel et nature humaine", in Terrain, n 40, 2003, p. 6.

5. Lenclud Gérard, "La tradition n'est plus ce qu'elle était...", in Terrain, n 9, 1987, pp. 110-123.

6. Lioré Julie, Les enfants et les adolescents d'origine ouest-africaine à Marseille. Des identités en construction, Sarrebruck,

Éditions universitaires européennes, 2010.

7. Seuls les interlocuteurs et les extraits d'entretien issus de mon terrain de thèse seront précisés (par "terrain 1998/2001"). Sans précision, les autres seront à associer au terrain plus récent (2008/2010).

8. Comportements alimentaires des adolescents et origines culturelles : "Étude comparée du rapport à la nourriture des jeunes de 12 à 19 ans, PACA/Alsace" (Programme de recherche OCHA-Cniel/UMR 6578 et 7043.

9. Quiminal Catherine, "La famille soninké en France", Hommes \& Migrations, n¹185, 1995, p. 26.

10. Toutes les fêtes ou pratiques dites "traditionnelles" ne doivent pas être entendues comme étant figées ni uniformes, mais sans cesse recomposées (que ce soit dans le pays d'origine comme en situation de migration), dynamiques et mouvantes par une pluralité d'influences.

11. Les filles seront simplement appelées par leur prénom, tandis que les mères seront distinguées par la mention

"Madame" suivie de leur prénom. Les prénoms ont été modifiés en respectant l'appartenance ethnique ou religieuse.

12. Barou Jacques, Verhoeven Moniel, "Alimentation et rôles familiaux. La cuisine familiale des immigrés africains", Ethnologie française, vol. XXVII, 1997, p. 96.

13. Rares sont les foyers visités à posséder des livres de recettes. Le rapport à l'écrit des parents résulte de paramètres sociologiques, comme le niveau d'instruction ou la situation socioprofessionnelle, et non de critères strictement culturels ou religieux comme le pays d'origine, l'appartenance ethnique ou la confession religieuse.

14. Choron-Baix Catherine,"Transmettre et perpétuer aujourd'hui", op. cit., p. 357.

15. Erny Pierre, L'enfant et son milieu en Afrique noire, Paris, L'Harmattan, 1987, p. 83.

16. Idem, p. 130.

17. Idem, p. 131.

18. Erny Pierre, Ethnologie de l'éducation, Paris, L'Harmattan, 1991 (1ère éd. PUF 1981), p. 163.

19. Sauces : mafé (pâte d'arachide), yassa (poulet, oignons en grande quantité et citron) + riz ; Plat : tieboudien (poisson farci, légumes : carottes, chou, igname, djaratou) + riz.

20. Crenn Catherine, "Le tajine à la bouillie bordelaise. Transmission des manières de cuisiner dans les familles des ouvriers agricoles immigrées dans le vignoble bordelais", in Cahiers de l'OCHA, n 11 : Faire la cuisine, 2006, p. 157.

21. Formule récurrente dans le discours des mères, plus rarement dans celui des filles.

22. Chrétien Jean-Pierre, La dimension historique des pratiques alimentaires en Afrique, in Diogène, $\mathrm{n}^{\circ} 144$, 1988 , pp. 95.

23. Constat fait auprès des adolescents d'origine étrangère toutes origines confondues (AlimAdos).

24. Maire Bernard, "Que savons-nous de l'alimentation des migrants?", in Lettre scientifique IFN, n ${ }^{\circ} 129,2008$, p. 4.

25. Crenn Catherine, "Le tajine à la bouillie bordelaise. Transmission des manières de cuisiner dans les familles des ouvriers agricoles immigrées dans le vignoble bordelais", op. cit., p. 159. 
